\title{
Im Alter geht es um den gelungenen Alltag
}

Die alternde Bevölkerung stellt die Medizin vor neue Probleme. Es geht weniger um das Kurieren, sondern vielmehr darum, den Alltag gelungen zu meistern. Bei diesem ganzheitlichen Ansatz kommt der Pflege eine wichtige Rolle zu, von der Ärztinnen und Ärzte profitieren. Prof. Dr. Lorenz Imhof, Professor für Pflegewissenschaften an der Zürcher Hochschule für angewandte Wissenschaften (ZHAW) in Winterthur, forscht über die Auswirkungen des demografischen Wandels und dessen Konsequenzen.

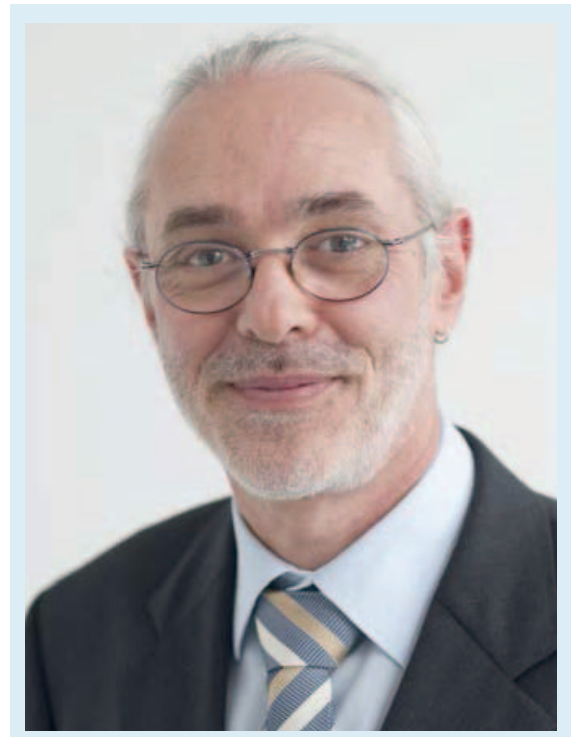

Gut leben im Alter

Wie der medizinische Alltag aussehen wird, wenn die Babyboomer betagt sind, zeichnet sich am Horizont bisher nur schemenhaft ab. Die Gesundheitsversorgung wird sich ändern, weil immer weniger Ärzte auf dem Land Dienst tun, ständig auf die Kosten geschaut wird und die Bedürfnisse von chronisch kranken Menschen andere sind als jene von jungen Menschen, die nur vorübergehend krank sind.
Die Babyboomer kommen in die Jahre und bald werden mehr Menschen alt sein als jemals zuvor in der Schweiz. Welche Auswirkungen wird es für die Medizin und die Pflege haben, wenn immer mehr Menschen immer älter und bedürftiger werden?

Prof. Dr. Lorenz Imhof: Zum einen sind alte Menschen häufig chronisch krank. Das bedeutet, dass sie Leiden haben, die sie im Alltag direkter und langfristiger fordern, als das in anderen Lebensphasen der Fall gewesen ist. Zum anderen sind ihre Möglichkeiten, Einschränkungen zu kompensieren, reduziert. Wenn man älter ist, kann man auf funktionale Körperveränderungen weniger gut reagieren. Das ist eigentlich die grosse Herausforderung im Alter: Wie gehe ich mit einer Gesundheitssituation um, die mir den Alltag immer beschwerlicher macht. In solchen Situationen nimmt die Bedeutung von professioneller Hilfe durch Pflegefachpersonen und Ärzte ebenso zu wie der Unterstützungsbedarf durch Familienangehörige.

In jüngeren Jahren kann man die Folgen gesundheitlicher Einschränkungen durch Umstellungen des Alltags oder durch Änderungen der Lebensweise auffangen?
Ein einfaches Beispiel: Wenn man sich als junger Mensch ein Bein gebrochen hat, hüpft man mit Krücken halt auf dem anderen. Bei einer Person, die 80 Jahre alt ist, geht das nicht mehr so einfach. Die Reserven sind viel kleiner, etwas zu kompensieren. Im Alter verändern sich auch die Denkprozesse. Ältere Menschen denken langsamer und gemächlicher. Anderseits darf man nicht unterschätzen, dass bestimmte kognitive Möglichkeiten zunehmen. Heute wissen wir, dass viele neuronale Vernetzungen erst ab etwa dem 45. Lebensjahr gebildet werden. Diese Vernetzungen sorgen dafür, dass man weniger eindimensional denkt, komplexe Situationen besser erfasst und sich damit auch kreative Kompensationsmöglichkeiten entwickeln lassen.

Der Umgang mit Krankheit im Alter hat auch etwas mit der Einstellung, mit einer gewissen philosophischen Lebensführung zu tun?

Im Prinzip schon, nur ist es schwierig, erst im Alter damit beginnen zu wollen, sich mit der richtigen Einstellung den Beschwerden des Alters $\mathrm{zu}$ stellen. Menschen sind $\mathrm{zu}$ den Persönlichkeiten geworden, die sie sind, weil sie im Laufe ihres Lebens 
bestimmte Entscheidungen getroffen haben. Die Haltung im und zum Alter ist ein Produkt ihres ganzen Lebens.

Das heisst, dass man sich schon in jungen Jahren mental darauf vorbereiten sollte, dass man einmal alt sein wird?

Ich bin skeptisch, dass Menschen sich auf das Alter vorbereiten können. Man kann sich vielleicht auf das Altern vorbereiten, aber nicht auf das Alter. Ich glaube nicht, dass sich jemand, der 30 oder 20 Jahre alt ist, vorstellen kann, wie der Alltag mit 70 Jahren ist. Vielleicht hilft es, dass Menschen früh damit beginnen, sich Zeit zu nehmen, über das eigene Tun nachzudenken. Wer sich nicht nur am Kick des Moments berauscht, sondern Veränderungen bewusst wahrnimmt, wird vom Alter nicht überrascht und schafft sich ein Repertoire, mit herausfordernden Veränderungen bis ins hohe Alter umzugehen.

\section{Sie plädieren dafür, die Lebens-} führung der Altersstufe anzupassen und nicht zu versuchen, seiner Jugend bis zum Stadium der Altersund Hinterlassenenversicherung nachzurennen?

Es gibt Untersuchungen, die Hinweise liefern, dass es eine grosse Bedeutung hat, sein Alter «anzunehmen». In meiner Studie zur Vergesslichkeit im Alter hat sich gezeigt, dass Menschen besser mit der Vergesslichkeit zurechtkommen, wenn sie sich z.B. sagen: «Es gehört halt zum Alter, dass ich Sachen vergesse.» Menschen, die akzeptierten, dass sie in eine neue Lebensphase eintraten, zu der auch Vergesslichkeit gehört, hatten deutlich mehr Ressourcen zur Verfügung. Sie suchten kreativer nach Möglichkeiten und erhielten bedeutend mehr Unterstützung von ihrem sozialen
Prof. Dr. Lorenz Imhof ist diplomierter Pflegefachmann und seit 2006 Forschungsleiter im Institut für Pflege am Departement Gesundheit der Zürcher Hochschule für angewandte Wissenschaften (ZHAW). 2011 hat ihn der Fachhochschulrat der ZHAW zum Professor ZFH (Zürcher Fachhochschulen) ernannt. Seine Forschungsschwerpunkte liegen unter anderem in der gemeindenahen integrierten Versorgung und in der gerontologischen Pflege.

Literaturhinweis: Imhof L, et al: Effects of an advanced practice nurse in-home health consultation program for communitydwelling persons aged 80 and older. J Am Geriatr Soc 2012;60:2223-2231.

Umfeld. Wenn jemand sagt, ich werde halt alt, entspannt das Situationen, in denen Dinge nicht mehr so klappen wie früher.

\section{Stammt die Ungeduld mit Alten von der jungen Generation oder eher von denjenigen, die auch schon fast im Seniorenalter sind?}

Ich denke, dass die Ungeduld ein Phänomen unserer heutigen Gesellschaft darstellt. Es ist die Hetze, die unser Leben bestimmt und die plötzlich nicht mehr zu dem passt, was Menschen im Alter an Geschwindigkeit vertragen. In den USA habe ich eine Studie zum Zeitempfinden von Parkinson-Patienten gemacht. Es hat sich gezeigt, dass diese Leute ihre eigene Geschwindigkeit hatten. Ihre Bewegungen hatten eine eigene Zeitlichkeit. Wenn Pflegefachpersonen diese nicht berücksichtigten, war Hilfe kaum möglich. Die Patienten konnten nicht aufstehen oder sich nicht anziehen, weil der Rhythmus dieser Unterstützung nicht dem körperlichen Zeitrhythmus der Menschen entsprach. Wenn Pflegefachpersonen den Rhythmus anpassten, konnten Parkinson-Patienten aber viel mehr Alltagshandlungen selbstständig ausführen.
Die Rücksicht auf das Zeitgefühl der Patienten hat in den Spitälern heute kaum noch Platz. Dort herrscht die Organisationszeit, die sich an der Uhr orientiert. Ärzte und Pflegefachpersonen haben kaum die Möglichkeit, sich an die Zeit der Menschen anzupassen, die sie behandeln und pflegen. Wir sind immer mehr in diesem Fünf-Minuten-Takt eingespannt, der einfach nicht dem Leben der Menschen entspricht, für die wir Verantwortung tragen. Die Ökonomisierung des Gesundheitswesens spielt da eine verhängnisvolle Rolle.

\section{Sie haben mit Spitex Plus eine Studie vorgelegt, in der Sie zeigen, dass Fachgespräche mit Betagten und deren Angehörigen helfen, die Gesundheitssituation zu verbessern oder zu stabilisieren. Sind entsprechende Programme eine gute Präventionsmöglichkeit?}

Auf alle Fälle. Der Einsatz von Pflegeexpertinnen (Advanced Practice Nurses) hat sich bewährt. Die Resultate der Studie stiessen international auf grosse Beachtung. Wir haben gezeigt, dass sich mit einer solchen Intervention unter dem Strich die Gesundheitskosten reduzieren liessen. Betagte mussten weniger häufig ins Spital, hatten weniger akute Gesundheitsprobleme oder stürzten seltener. Aber die Umsetzung in der Praxis muss nun die Hürde der Ökonomisierung nehmen und könnte daran scheitern. Denn am Anfang steht die Frage nach der Finanzierung. Weil ein solches Projekt etwas kostet, ist die Diskussion schwierig. In den angelsächsischen Ländern oder dort, wo eine geringe Ärztedichte vorhanden ist, sind solche Präventionsmodelle verbreiteter. Der Mangel an Fachärzten hat z.B. in den USA dazu geführt, dass die Pflege in diese Lücke gesprungen ist; so entstand der Beruf der Advanced Practice Nurse. Ich nehme an, dass die Bedeutung von gut ausgebildeten Pflegefach- 
personen in der Versorgung von Betagten in Zukunft zunehmen wird. Damit z.B. die spitalexterne Pflege (Spitex) ihre Aufgabe wahrnehmen kann, braucht es speziell ausgebildete Pflegeexpertinnen. Die chronischen Krankheiten nehmen zu, die Komplexität der Versorgung wird höher, und Patienten werden früher aus dem Spital entlassen und müssen dann zu Hause betreut werden. Die Rolle der Pflegefachpersonen ist heute viel anspruchsvoller als noch vor 20 Jahren. Dem versucht die Ausbildung mit dem klinisch ausgerichteten Masterstudiengang "Advanced Practice Nursing» (APN) gerecht zu werden.

\section{Was ist nötig, um die Versorgung der immer älter werdenden Bevölkerung in Zukunft auf sichere Beine zu stellen?}

Unsere Berechnungen zeigen, dass wir etwa $10 \%$ hochqualifizierte Pflegeexpertinnen mit einer APN-Ausbildung in der Spitex-Versorgung bräuchten. Dann würden wir wesentlich effizienter und besser arbeiten. Gemessen an dem, was man in der Schweiz sonst für alle möglichen Dinge ausgibt, sind die Kosten gar nicht so hoch, dies zu realisieren. Ein guter flächendeckender Standard in der ganzen Schweiz würde uns etwa so viel kosten, wie die Eidgenossenschaft jedes Jahr zur Unterstützung des Käseverkaufs im Ausland aufwendet.

Basierend auf unseren Studien sind wir optimistisch, dass sich der Erfolg rasch einstellen würde und die Zahl der Heimeintritte abnehmen könnte. Mit einer guten ambulanten pflegerischen Unterstützung und Beratung nehmen etwa die Zahl der Stürze und damit auch die Spitalaufenthalte ab. Denn Stürze sind ein wichtiger Risikofaktor für einen Heimeintritt. Ein Ausbau der Spitex würde die Möglichkeit schaffen, die pflegenden Angehörigen zu unterstützen. Man muss sich vor Augen halten, dass sie meist ebenfalls älter sind, wenn sie ihre Partnerinnen/ Partner oder Eltern der Generation 80 plus betreuen. Deshalb ist es sehr wichtig, ihre Gesundheit zu erhalten. Jahrelang Pflege zu leisten, ist anstrengend und manchmal auch ein Gesundheitsrisiko für körperliche Leiden bis hin zur Erschöpfung oder Altersdepression.

\section{Wo fühlen sich alte Menschen besser?}

Die meisten wollen zu Hause sein, und in der Schweiz sind die meisten auch noch in den eigenen vier Wänden. Auch bei den über 90-Jährigen lebt noch über die Hälfte zu Hause. Studien zeigen, dass Einsamkeit oder fehlende soziale Kontakte sowohl im Pflegeheim als auch zu Hause ein Problem sein können. Da spielt der Ort keine Rolle. Entscheidend ist, dass die Umgebung es erlaubt, sein Leben selbst zu gestalten, wie man das gewohnt war. Deshalb ist der Übertritt in ein Altersheim für viele Menschen ein grosses Problem, mit vielen Ängsten verbunden und eines der dramatischsten Lebensereignisse. Auch mit Unterstützung und bei besten Bedingungen geben sie ihr altes Leben auf und müssen sich einen neuen Alltag schaffen. Das ist im Alter sicher nicht einfach.
Welche Rolle könnten denn «neue» Wohnformen im Alter spielen? Die Idee von Alters-WGs, in denen Freunde gemeinsam alt werden, taucht ja öfters in der Diskussion auf.

Dass dies in der Praxis in der gleichen Wohnung mit gemeinsamer Küche und Badezimmer funktioniert, glaube ich weniger, obwohl nun die 68erGeneration in das Alter kommt, die mehr WG-Erfahrung hat. In einem Haus gemeinsam in getrennten Wohnungen zu leben - das könnte gehen. Dann hat jeder seinen Ort zum Rückzug, lebt gemeinsam und hat soziale Kontakte und Unterstützung. Der Vorteil liegt auf der Hand. Es bildet sich ein enges soziales Netz im Rahmen eines Hauses. Das könnte eine Möglichkeit sein.

Womit sich viele Menschen im Alter schwertun, sind Umstellungen ihrer Alltagsroutine. Gewohnheiten zu ändern, sich auf neue Abläufe einzustellen, verlangt enorme Flexibilität, die geübt sein will. Gerade das Zusammenbrechen der Routine ist für Menschen mit chronischen Krankheiten eine der zentralen Herausforderungen. Man merkt, dass etwas, was man 20 Jahre lang so gemacht hat, nicht mehr geht und man sich ändern und eine neue Routine kreieren muss. Wenn vieles nicht mehr so wie früher geht, setzt das die

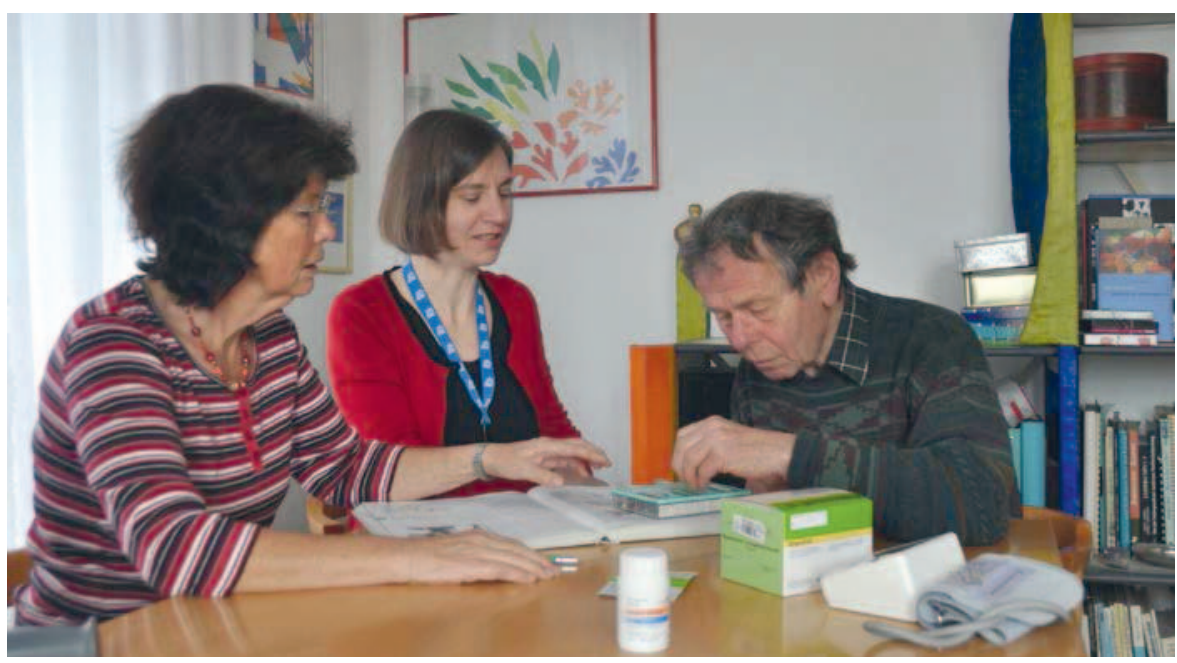

Abb. Die Betreuung durch Advanced Practice Nurses verbessert die Lebensqualität von Betagten. 
Menschen den ganzen Tag lang unter permanenten Druck. Das gilt nicht nur im Alter. Ein paar Beispiele, die ich während unserer Forschungsprojekte gehört habe: Alte Menschen, die dreimal pro Tag einkaufen, weil sie die Kommissionen ständig vergessen oder weil sie die Taschen mit allen Einkäufen nicht mehr auf einmal tragen können. Ein Ehepaar, bei dem die Frau alle Dinge am gleichen Ort haben möchte, weil sie sonst vergisst, wo sie sie hingelegt hat, der Mann das aber nicht tut. Das führt im $\mathrm{Zu}$ sammenleben dann dauernd zu Konflikten und Stresssituationen. Wir alle wissen, wie das ist, wenn man etwas braucht und es nicht findet. Wenn Betagten das häufig passiert, wird das Leben extrem aufwendig und unberechenbar. Menschen in solchen Situationen vermeiden den Stress, indem sie sich aus Beziehungen und gemeinsamen Aktivitäten zurückziehen.

\section{Gibt es Stressinterventions-} programme, mit denen älteren Menschen geholfen werden kann, mit diesen Situationen umzugehen?

Die Pflege hat die Idee, dass dies gelingt, wenn sie sich um den Alltag kümmert, wenn Pflegefachpersonen sich gemeinsam mit den Menschen für einen «gelingenden» Alltag einsetzen. Ich verwende diesen Begriff sehr gerne, bei dem es darum geht, dass alle Beteiligten etwas gemeinsam neu so planen, dass es funktioniert. So z.B. in unserem Spitex-PlusProjekt. Dort sprechen die Betagten und deren Angehörige mit den Pflegenden über ein Thema, das sie selbst bestimmen und das auf den Nägeln brennt. Wenn Schmerzen chronisch werden und deshalb den Alltag beherrschen, dann wird der Alltag zum Problem. Pflegefachpersonen versuchen deshalb, gemeinsam mit den Patientinnen neue Ideen $\mathrm{zu}$ entwickeln, wie man mit diesen Schmerzen trotzdem einen gelingenden Alltag kreieren kann. Dazu braucht die Pflegefachperson fundierte medizinische Kenntnisse, die Fähigkeit, eine Situation ganzheitlich zu erfassen, soziale Kompetenzen und natürlich viel Berufs- und Lebenserfahrung.

\section{In der Pflege wechseln oft die \\ Personen. Ist das ein Problem?}

Auf alle Fälle, denn die Pflegefachpersonen kommen ja ins Haus und betreten die Privatsphäre. Gute Pflege braucht dazu die Erlaubnis der Menschen. Das kann schnell gehen, aber manchmal dauert es Wochen, bis Unterstützung akzeptiert und Vertrauen aufgebaut ist. Im Zentrum der Pflege steht das «Sich-Sorgen» um Menschen. Dazu braucht es eine pflegerische, professionelle Beziehung. Sie ist das Arbeitsinstrument und kann nur geschaffen werden, wenn Pflegefachpersonen dafür die nötige Zeit erhalten. Konstante Beziehungen sind wichtig, und wenn jeden Tag eine neue Pflegefachkraft vorbeikommt, gelingen diese nicht. Das hat Konsequenzen für die Qualität der Versorgung von pflegeabhängigen Personen. In einer Studie zum begleiteten Suizid in der Schweiz beklagte sich ein betagter Patient darüber, dass er in den letzten 2 Jahren täglich von neuen Pflegepersonen betreut wurde. «Das reicht jetzt, das schaffe ich nicht mehr", war sein Fazit in seiner Erklärung zum Suizidwunsch.

\section{Der Pflege kommt eine zentrale Bedeutung bei der Versorgung der älteren Generationen zu?}

Das ist so, und es gibt gute Beispiele, wie sie ihre Verantwortung heute schon exzellent wahrnimmt. Zum Beispiel in der Palliativpflege. Fachstellen, besetzt mit spezialisierten Pflegefachpersonen, die bereits heute Konstanz und die Verfügbarkeit garantieren und so eine enorme Bedeu- tung erhalten, wenn es darum geht, dass Betagte ein gutes Leben trotz chronischer Leiden führen können. «Gut» bedeutet dabei, dass der Alltag gemeistert werden und auch noch lustvoll sein kann.

\section{Welche Rolle spielt eigentlich ein Hausarzt noch in dieser Phase?}

Wir sehen die Pflegeexpertin als so etwas wie die Fallmanagerin, wobei es sich aber um Menschen und nicht um Fälle handelt. Sie ist in der Wohnung, schaut, ob alles vorhanden ist, was es zur Versorgung des Patienten braucht, spricht mit Angehörigen, überprüft die Medikamente, beobachtet deren Wirkung und kümmert sich um zusätzliche Dienste, die eingeschaltet werden müssten. Sie bildet ein Netz integrierter Versorgung. Wenn alles läuft, ist der Hausarzt entlastet. Er hat jemanden, der ihm genau sagen kann, ob etwa die Therapie anschlägt, und der auch fundierte medizinische Beurteilungen machen kann. Er muss bei Bedarf nur noch mit einer Person telefonieren, die dann verantwortlich notwendige Schritte selbstständig in die Wege leitet. Unsere Umfragen bei Hausärzten zeigen, dass dieses Modell für den Arzt oft einfacher und effizienter ist und bessere Ergebnisse erzielt. Wo das Zusammenspiel gut ist, ist das eine tolle Zusammenarbeit, von der alle profitieren.

\section{Wie wichtig wird zukünftig die Familie bei der Betreuung und Pflege von älteren Menschen sein?}

Wie heute schon wird die Familie auch in Zukunft enorm wichtig für ältere Menschen sein. Der Unterstützungsaufwand, den Angehörige betreiben, ist riesig. Auch wenn es keine Grossfamilien mehr gibt, sondern die Menschen in Zweierbeziehungen oder alleine leben, sind Angehörige oft örtlich sehr nahe 
und dadurch in kürzester Zeit verfügbar. Doch das Bild vom alten Menschen, der Hilfe benötigt, ist falsch. Wenn jemand heute mit 65 Jahren in Pension geht, hilft er noch 10 Jahre lang z.B. beim Enkelhüten mit und unterstützt die eigenen Kinder mit verschiedenen «Dienstleistungen». Dadurch stützen ältere Menschen durch Freiwilligenarbeit in sozialen Netzen die bestehenden Sozialstrukturen.

Altern heisst nicht, primär pflegebedürftig zu sein. Wenn heute Menschen länger leben, dann tun sie dies auch in guter Gesundheit. Pflegebedürftigkeit und Pflegeheimeintritte finden heute später statt als noch vor 20 Jahren. Ältere Menschen sind deshalb auch eine Ressource und ein wichtiger Teil unserer Gesellschaft. Das bedeutet aber auch, dass die Gesellschaft ihre Verantwortung wahrnehmen muss, wenn diese Menschen professionelle Pflege benötigen. Pflegefachpersonen sind dazu da, diese gesellschaftliche Verpflichtung in die Tat umzusetzen.

Interview: Oliver Klaffke 